\title{
Bloodstream Infection Etiology among Children and Adults
}

\author{
Svetlana I. Kolesnichenko $\left(\mathbb{D}\right.$, Alyona V. Lavrinenko $\mathbb{D}^{D}$, and Lyudmila L. Akhmaltdinova
}

Shared Resource Laboratory, Medical University of Karaganda, Karaganda 100008, Kazakhstan

Correspondence should be addressed to Svetlana I. Kolesnichenko; kolesnichenko@kgmu.kz

Received 3 November 2020; Accepted 12 February 2021; Published 1 March 2021

Academic Editor: Luigi Santacroce

Copyright (C) 2021 Svetlana I. Kolesnichenko et al. This is an open access article distributed under the Creative Commons Attribution License, which permits unrestricted use, distribution, and reproduction in any medium, provided the original work is properly cited.

\begin{abstract}
Every year in the world, sepsis occurs in 31.5 million people, and the number of deaths reaches 5.3 million per year. There are not enough studies that describe etiological structure of sepsis pathogens in different groups of population of the Republic of Kazakhstan. In this study, we have investigated difference of local sepsis etiology and antibiotic susceptibility among children and adults. A total 200 blood samples were examined using the standard and express method of identification of bloodstream pathogens. The determination of antimicrobial sensitivity was carried out by the disc-diffusion method according to CLSI guidelines. Overall, 23/90 (25.5\%) positive blood cultures were isolated from adult patients and 43/110 (39\%) from pediatric patients. It was found that children are statistically more often affected with bacterial bloodstream infection than adults $(p<0.05)$. The Gram-positive bacteria are the leading cause of sepsis in both groups: S. epidermidis (35.5\%) in pediatric patients and S. aureus $(21.7 \%)$ in adults. However, statistical significance was detected in pediatric patients $(p<0.05)$. The number of resistant strains of S. epidermidis (MRSE) in the group of children was $66.7 \%$, while in adults, all S. epidermidis was resistant to azithromycin and cefoxitin (MRSE). S. aureus strains from adult patients and children had a similar picture of antibiotic patterns. The proportion of MRSA in pediatric patients was $16,6 \%$, and in adult patients, $20 \%$. Enterobacterales (39\%) were the second cause of sepsis in adult patients. $62.5 \%$ of Enterobacterales strains isolated from adults were phenotypically identified as ESBL, while in pediatric patients, $25 \%$ of ESBL producers were isolated. We have noted the resistance to antibiotics that are prescribed according to protocols of treatment of the Republic of Kazakhstan in the strains isolated from the patient's blood.
\end{abstract}

\section{Introduction}

Sepsis is a life-threatening organ dysfunction which occurs as a result of a body's response to infection causes [1]. Every year in the world, sepsis occurs in 31.5 million people, and the number of deaths reaches 5.3 million per year [2]. To date, sepsis remains the main cause of mortality in children under 1 year of age. According to the WHO, about 3 million cases of sepsis among newborns and 1.2 million among children are detected annually in the world. The mortality rate from sepsis varies from $11 \%$ to $19 \%$ per 100,000 live births [3]. In the United States, for 72,000 hospitalized children diagnosed with sepsis, the mortality rate is $25 \%$ [4]. According to the SPROUT study, in 2013-2014, complications associated with sepsis in children ranged from $6 \%$ to $20 \%$ in Europe, South and North America, Australia/New Zealand, and Africa [5].
The primary foci of bloodstream infections are more often the lower respiratory tract in general [6], the respiratory system (40\%), and the circulatory system (19\%) in the pediatric departments [5]. According to Daneman et al.'s study [7], the most common sources of bacteremia in adults were the lung (27\%), abdomen $(25 \%)$, and urinary tract (23\%).

Previous studies have found that the leading role in the bloodstream infections in adults belongs to microorganisms of the Enterobacterales family (Escherichia coli and Klebsiella spp.) and Enterococcus spp. [7]. If we talk about bloodstream infections in children, the data vary, according to a YaleNew Haven Hospital study for 2004-2013, E. coli was the cause of early sepsis in newborns in $45 \%$ of cases, followed by group B Streptococcus (36\%) [8], and a similar situation is described by researchers from the United States $[9,10]$. Other studies show that the leading positions are occupied 
by Gram-positive bacteria, coagulase-negative staphylococci (CoNS), S. aureus and E. faecalis (13\%) [8]. In studies conducted in Southeast Asia, China, an equal distribution of Gram-positive and Gram-negative microorganisms in the etiology of blood stream infections in adults was noted, and Gram-positive pathogens dominated in children [11].

There are not much data on sepsis in the countries of the former Soviet Union: the epidemiology of sepsis in Russia can be judged from the Rudnov et al. multicenter study [6], where the prevalence of bloodstream infections in ICU was $34.1 \%$. In the Republic of Kazakhstan, there are only a few local data, which do not reflect the entire epidemiological situation $[12,13]$.

Over the past 10 years, the advent of new technologies in microbiology has improved the diagnosis of septic conditions. The use of the direct detection method, using matrix laser desorption ionization time-of-flight mass spectrometry-MALDI-TOF, has become available for many laboratories. The simplicity of the preparation samples, the high quality of identification, time saving, and the low cost of analysis have made this method popular [14].

\section{Objective}

The objective of this study is to investigate difference of local sepsis etiology and antibiotic susceptibility among children and adults.

\section{Materials and Methods}

The study was conducted on the basis of the shared resource laboratory of the Medical University of Karaganda (Kazakhstan). Material for the study was blood samples from 200 patients diagnosed with sepsis. Patients were divided into 2 groups by age: children from 0 to 18 years old and adults from 18 to 99 years old. The average age of infants was $3.15 \pm 2.49$ months, and in children, $5.7 \pm 5.3$ years; in the group of adult patients, the average age was $51.6 \pm 17.8$ years (Table 1). Peripheral blood sampling was carried out directly in intensive care units from patients with aseptic technique in BD BACTEC ${ }^{\mathrm{TM}}$ Peds Plus vials before the beginning of antibiotic therapy. Blood was taken in a volume of 1-2 $\mathrm{ml}$ in children and adults. Within an hour from the time of blood sampling, the vials were delivered to the laboratory for further incubation using the BD BACTEC ${ }^{\text {тм }}$ FX blood culture system.

When a signal about a positive sample appears, $5 \mathrm{ml}$ of broth is taken into serum gel-separator tubes. Gel-separator tubes were centrifuged on $3000 \mathrm{rpm}$ for 10 minutes to separate media and bacteria from erythrocytes. Then, supernatant is discharged, and a thin white layer above gel is carefully mixed in 1-1,5 ml ultrapure water, and moved into a 1, $5 \mathrm{ml}$ Eppendorf tube. This sample is being centrifuged for 2 minutes on $13000 \mathrm{rpm}$. Supernatant is discharged, and the pellet is applied on target [15] and identified using the MALDI-TOF Microflex mass spectrometer (Bruker Daltonics, Germany). Identification was considered successful at the level of species with a high degree of confidence when the score exceeded 2.0; if the score was between 2.0 and 1.7, identification was considered successful at the genus level with adequate confidence [16].

Simultaneously, positive blood culture samples were cultured on blood agar with horse blood for 18-20 hours to exclude mixed cultures and to get isolated colonies for the antibiotic susceptibility test.

The determination of antimicrobial sensitivity was carried out for the prevailing microorganisms for each group of patients. Susceptibility of bacterial isolates to antibacterial drugs was carried out by the disk diffusion method to following antibiotics: penicillin $\mathrm{G}(10 \mathrm{u})$, ampicillin $(10 \mu \mathrm{g})$, cefepime $(30 \mu \mathrm{g})$, imipenem $(10 \mu \mathrm{g})$, meropenem $(10 \mu \mathrm{g})$, amikacin $(30 \mu \mathrm{g})$, gentamycin $(10 \mu \mathrm{g})$, netilmicin $(30 \mu \mathrm{g})$, tobramycin $(10 \mu \mathrm{g})$, nalidixic acid $(30 \mu \mathrm{g})$, ciprofloxacin $(5 \mu \mathrm{g})$, levofloxacin $(5 \mu \mathrm{g})$, linezolid $(30 \mu \mathrm{g})$, clindamycin $(2 \mu \mathrm{g})$, azithromycin $(15 \mu \mathrm{g})$, vancomycin $(30 \mu \mathrm{g})$, teicoplanin $(30 \mu \mathrm{g})$, fusidic acid $(10 \mu \mathrm{g})$, and tetracycline $(30 \mu \mathrm{g})$. Analysis of the results was carried out in accordance with the recommendations of CLSI 2018 [17]. Determination of bacterial isolate susceptibility to colistin was carried out by the broth microdilution method in accordance with the EUCAST guidelines [18, 19].

Phenotypic detection of methicillin resistant S. aureus (MRSA) and S. epidermidis (MRSE) was conducted by cefoxitin disk $(30 \mu \mathrm{g})$. MRSA positive strains had zone of inhibition $\leq 23 \mathrm{~mm}$ [17].

ESBL producers were determined by using cefuroxime $(30 \mu \mathrm{g})$, amoxicillin/clavulanic acid $(20 / 10 \mu \mathrm{g})$, and cefotaxime $(30 \mu \mathrm{g})$ disks [17].

Internal quality control was carried out on the control strains: Staphylococcus aureus ATCC 25923, Pseudomonas aeruginosa ATCC 27853, Escherichia coli ATCC 25922 (ESBLand AmpC-), Klebsiella pneumoniae WHO-3 (ESBL +), and Enterobacter cloacae WHO-238 (AmpC + and ESBL-).

The analysis of susceptibility to antibacterial drugs was carried out by calculating the $95 \%$ confidence interval using the WHONET 5.6 program. Statistical analysis was carried out in the STATISTICA 7.0 program using the chi square.

\section{Results and Discussion}

Overall, a total 200 blood samples were processed. Of these, 23/90 (25.5\%) positive blood cultures were isolated from adult patients and $43 / 110(39 \%)$ from pediatric patients. It was found that bacteremia is statistically more often detected in children with sepsis than in adults $(p<0.05)$. From all positive samples, 4/66 (6\%) were represented as mixed cultures (the associations of two microorganisms). It is useful to note that three of them were from patients up to 1 month of life. However, there is no statistical significance between frequency of mixed isolates and age of participants $(p<0.05)$.

The primary foci of infection in the examined patients were distributed as follows: in adult patients, the most common source of infection was the blood-vascular system (78.2\%) and in pediatric patients, the respiratory system (48.6\%) (Table 2).

When examining the etiological structure of bloodstream infections, in the group of children, the most 
TABLE 1: Characterization of patients by groups.

\begin{tabular}{lcccc}
\hline Group of patients & Age & Mean age \pm SD & Total examined & Number of “+” samples \\
\hline \multirow{2}{*}{ Children } & $0-12$ month & $3.15 \pm 2.49$ & 57 & $43(39 \%)$ \\
& $1-18$ years & $5.7 \pm 5.3$ & 53 & $23(25.5 \%)$ \\
\hline Adults & $18-99$ & $51.6 \pm 17.8$ & 90 & 23 \\
\hline
\end{tabular}

TABle 2: Primary foci of infection in patients with sepsis.

\begin{tabular}{lcc}
\hline Primary foci & Children $(n=43)(\%)$ & Adults $(n=23)(\%)$ \\
\hline Respiratory tract & 48,6 & 13 \\
Blood-vascular system & - & 78,2 \\
Gastrointestinal tract & 23,2 & 4,3 \\
Skin-soft tissue/bone-joint & 9,3 & - \\
Intranatal & 11,6 & - \\
Central nervous system & 6,9 & - \\
Genital tract & - & 4,3 \\
\hline
\end{tabular}

prevalence microorganism was S. epidermidis (35.5\%) and in the group of adult patients, S. aureus (21.7\%) (see Figure 1 and Figure 2). The Gram-positive bacteria play the leading role in patients of both groups. However, the ratio of Grampositive and Gram-negative microorganisms in the children group was differed significantly: $37: 5$ (86\%:11.6\%), so the Gram-positive bacteria in children were detected statistically more often $(p<0.05)$. In adults, the ratio of Gram-positive and Gram-negative bacteria is not much different, 14:9 $(60.8 \%: 39.1 \%)$, and the second place after S. aureus in adults belongs to microorganisms of the Enterobacterales family (E. coli (13\%), K. pneumonia (8, 7\%), and Salmonella sp.(4, 3\%)) (see Figure 1).

The study identified 3 cases of candidemia, and all of them were found in children. The causative agents were C. albicans and C. parapsilosis. According to past studies, the role of Candida fungi in the development of BSI has been marked among newborns and children of the first year of live much more often than among adults [20, 21].

During testing of the prevailing pathogens to antibiotics, we did not find trends of antimicrobial resistance among groups. The S. epidermidis strains isolated from the blood of pediatric patients were completely susceptible to penicillin, 100\% (95\% CI 74.7-100), 68.8\% (95\% CI 41.5-87.9) cases were resistant to azithromycin, 33.3\% (95\% CI 13.0-61.3) cases were resistant to clindamycin, $35.7 \% \quad(95 \%$ CI 14.0-64.4) were resistant to ciprofloxacin, and 50\% (95\% CI 20.1-79.9) were resistant to levofloxacin; to the group of aminoglycosides (gentamicin), 18 were resistant, 8\% (95\% CI 5.0-46.4) (see Figure 3). The proportion of MRSE was $66.7 \%$ (95\% C.I. 38.7-87.0).

S. epidermidis, isolated from an adult group of patients, was characterized by a higher level of resistance to antibacterial drugs, and all strains were resistant to azithromycin and cefoxitin (MRSE). The remaining antibiogram data were similar to those in the group of children. All S. epidermidis strains isolated from adults and children were highly sensitive to vancomycin, linezolid, teicoplanin, and fusidic acid. The data obtained are consistent with the literature: in Amita Jain et al.'s study of catheter-associated bloodstream infections in the pediatric population, $63.1 \%$ oxacillin resistant CoNS and high glycopeptide sensitivity were obtained [22]; $66.6 \%$ of MRSE were found in a study of Santarpia et al. [23], but they also detected 55\% of vancomycin-resistant strains. In our study, no vancomycin-resistant strains were detected, so it can be used to treat BSI caused by Gram-positive pathogens.

An important role in the etiology of sepsis in adults in our study was played by $S$. aureus (21.7\%). S. aureus strains from adult patients and children had a similar picture of antibiotic patterns and were characterized by moderate sensitivity to antimicrobial agents. $1 / 5$ (20\%) of the strains from adults were MRSA; from pediatric patients, the proportion of MRSA was 1/6 (16.6\%).

Enterobacterales (39\%) were the second cause of sepsis in adult patients. $42.9 \%$ (95\% C.I. 11.8-79.8) strains were resistant to fluoroquinolones (ciprofloxacin) and tetracycline while those from pediatric patients were highly susceptible, 100\% (95\% CI 0.0-60.4). Colistin (MIC> 2 100\% (95\% CI 0.0-37.1) and imipenem had high antibacterial activity in both groups. Meropenem was less active in adults, $14.3 \%$ of resistant strains. $28.6 \%$ (95\% CI 5.1-69.8) of strains from adults were resistant to gentamicin, so as in children, $25 \%$ (95\% CI 1.3-78.1). Other aminoglycosides in pediatric patients were completely susceptible (100\%), while 12, 5\% (95\% CI 0.7-53.3), of isolates from adult patients were resistant to tobramycin and 14, 3\% (95\% CI 0.8-58.0), to netilmicin (see Figure 4). 62.5\% of Enterobacterales strains isolated from adults were phenotypically identified as ESBL, while in pediatric patients, were isolated $25 \%$ of ESBL producers. In a study by Abdallah H. M., 48.93\% of ESBL producers were isolated from patients with a bloodstream infection, which is consistent with our data [24]. Similar results were obtained in China, and $67.8 \%$ of ESBL producers were isolated from positive blood samples [25]. 


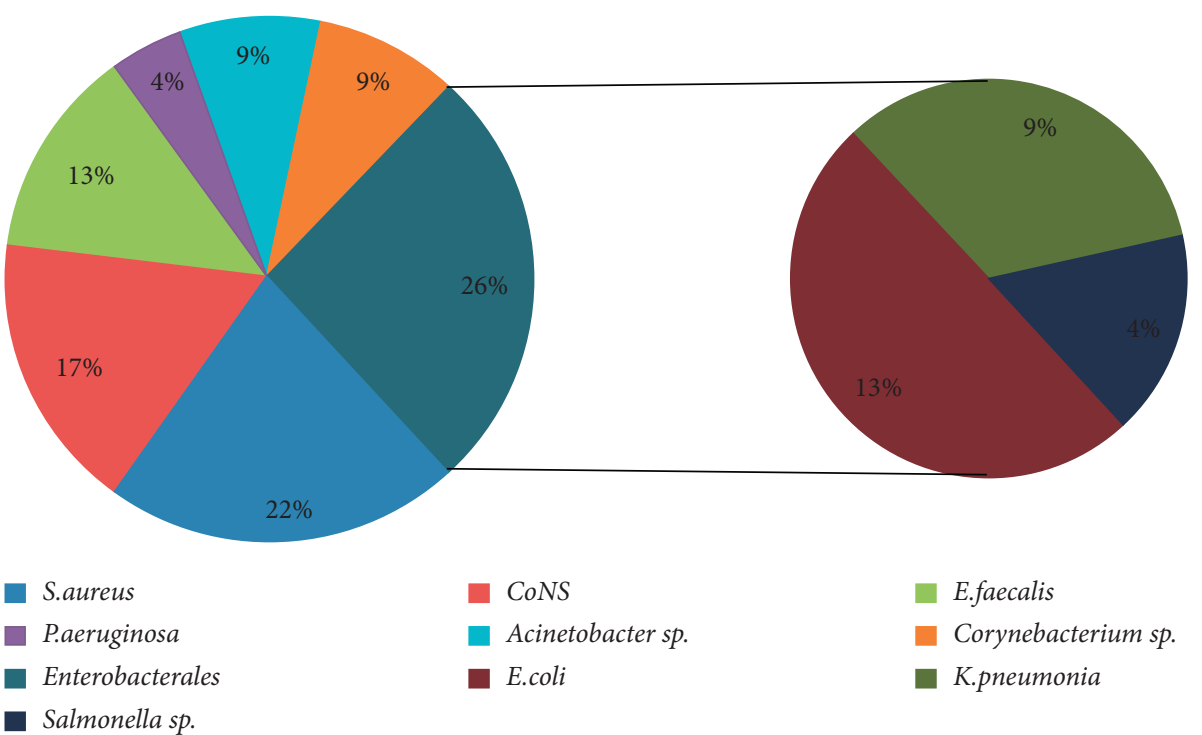

Figure 1: The causative agents of sepsis in adult patients.

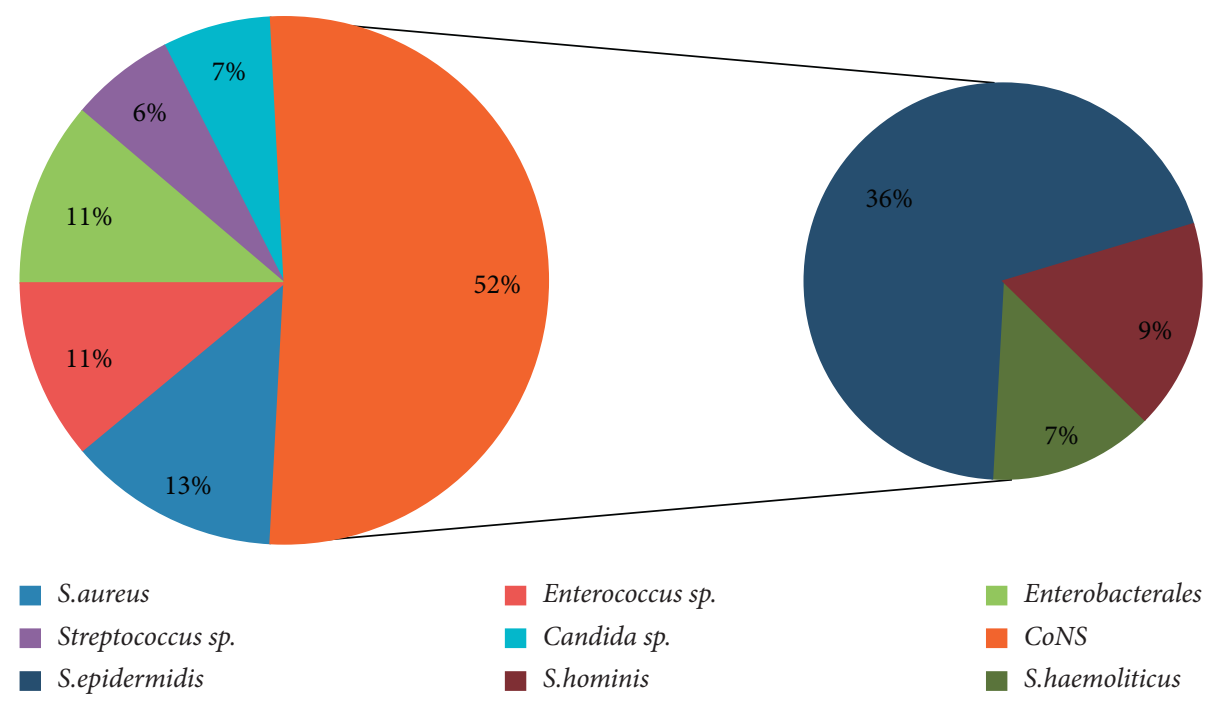

FIgURE 2: The causative agents of sepsis in children. 


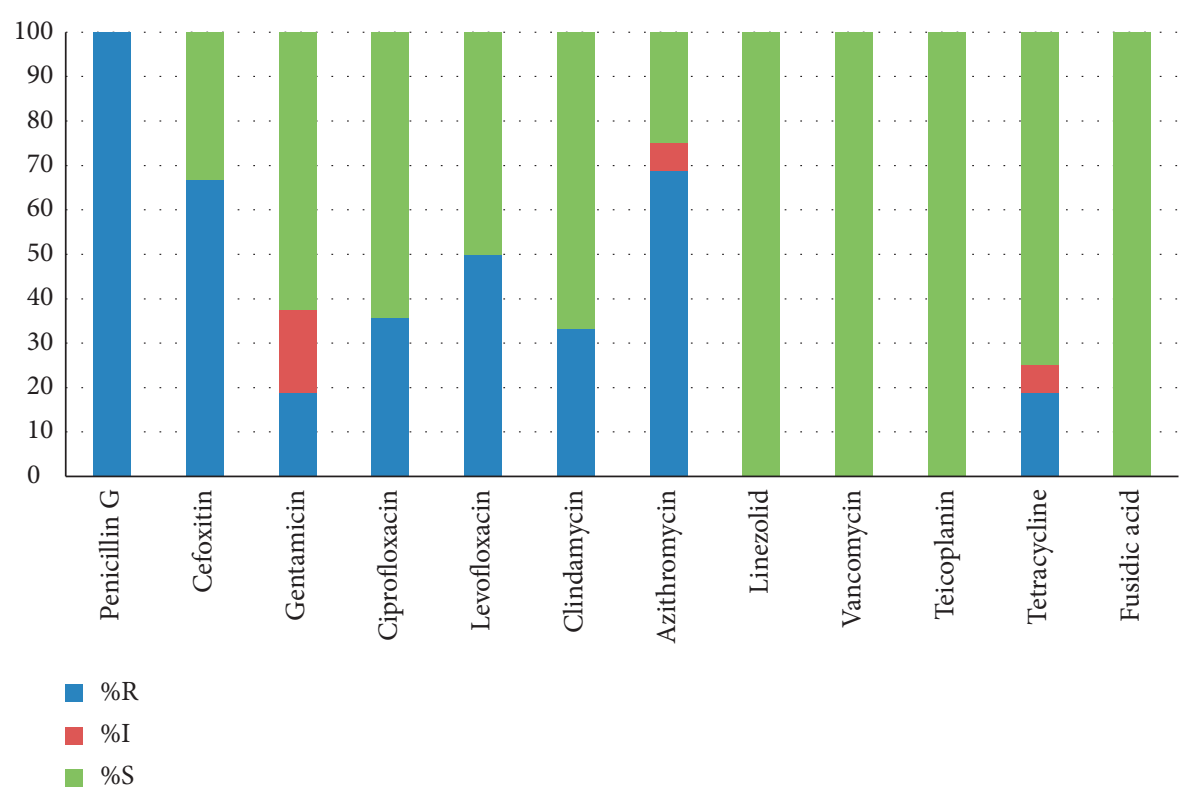

FIgURE 3: Sensitivity to antibiotics S. epidermidis from children.

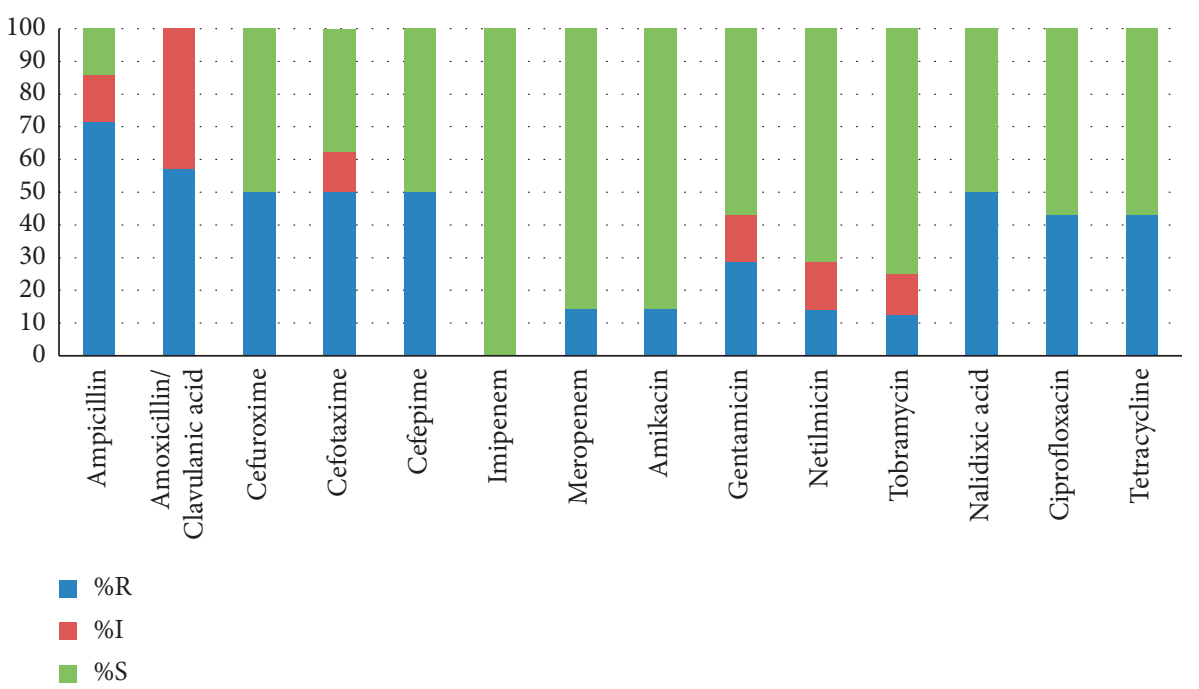

Figure 4: Sensitivity to antibiotic Enterobacterales strains from adults.

\section{Conclusions}

In the results of our study, we found that bacteremia is statistically more often detected in children with sepsis than in adults $(p<0.05)$. The most frequently isolated microorganisms in both groups were Gram-positive cocci: in children, S. epidermidis (35.5\%); in adults, S. aureus (21.7\%). The Gram-positive bacteria in children were detected statistically more often $(p<0.05)$ than Gram-negative with ratio $37: 5$ $(86 \%: 11.6 \%)$. The number of resistant strains of S. epidermidis (MRSE) in the group of children (66.7\%) significantly exceeds the number of those in adults, which may be due to the localization of the primary focus of infection and more frequent administration of antistaphylococcal drugs. In adult patients, a large percentage of ESBL (62.5\%) relative to children (25\%) draws attention, which is associated with the spectrum of prescribed antibacterial drugs according to the protocols of diagnosis and treatment of the Republic of Kazakhstan.

\section{Data Availability}

The data used to write this publication are the property of the Ministry of Education and Science of the Republic of Kazakhstan and are available from the corresponding author upon request.

\section{Conflicts of Interest}

The authors declare that there are no conflicts of interest regarding the publication of this paper. 


\section{Acknowledgments}

This research was funded by the Science Committee of the Ministry of Education and Science of the Republic of Kazakhstan (grant no. AP08857386).

\section{References}

[1] M. Singer, C. S. Deutschman, C. W. Seymour et al., "The third international consensus definitions for sepsis and septic shock (Sepsis-3)," JAMA, vol. 315, no. 8, pp. 801-810, 2016.

[2] C. Fleischmann, A. Scherag, N. K. J. Adhikari et al., "Assessment of global incidence and mortality of hospital-treated sepsis. Current estimates and limitations," American Journal of Respiratory and Critical Care Medicine, vol. 193, no. 3, pp. 259-272, 2016.

[3] C. Fleischmann-Struzek, D. M. Goldfarb, P. Schlattmann, L. J. Schlapbach, K. Reinhart, and N. Kissoon, "The global burden of paediatric and neonatal sepsis: a systematic review," The Lancet Respiratory Medicine, vol. 6, no. 3, pp. 223-230, 2018.

[4] A. Ruth, C. E. McCracken, J. D. Fortenberry, M. Hall, H. K. Simon, and K. B. Hebbar, "Pediatric severe sepsis," Pediatric Critical Care Medicine, vol. 15, no. 9, pp. 828-838, 2014.

[5] S. L. Weiss, J. C. Fitzgerald, J. Pappachan et al., "Global epidemiology of pediatric severe sepsis: the sepsis prevalence, outcomes, and therapies study," American Journal of Respiratory and Critical Care Medicine, vol. 191, no. 10, pp. 1147-1157, 2015.

[6] V. A. Rudnov, D. V. Belsky, A.V. Dekhnich, and the RIORITA study group, "Infections in Russian ICUs: results of the nationwide multicenter study," Clinical Microbiology and Antimicrobial Chemotherapy, vol. 13, no. 4, pp. 294-303, 2011.

[7] N. Daneman, A. H. Rishu, A. H. Pinto et al., "7 versus 14 days of antibiotic treatment for critically ill patients with bloodstream infection: a pilot randomized clinical trial," Trials, vol. 19, no. 1, p. 111, 2018.

[8] M. J. Bizzarro, V. Shabanova, R. S. Baltimore, L.-M. Dembry, R. A. Ehrenkranz, and P. G. Gallagher, "Neonatal sepsis 20042013: the rise and fall of coagulase-negative staphylococci," The Journal of Pediatrics, vol. 166, no. 5, pp. 1193-1199, 2015.

[9] E. J. Weston, T. Pondo, M. M. Lewis et al., "The burden of invasive early-onset neonatal sepsis in the United States, 20052008," Pediatric Infectious Disease Journal, vol. 30, no. 11, pp. 937-941, 2011.

[10] B. J. Stoll, N. I. Hansen, P. J. Sánchez et al., "Early onset neonatal sepsis: the burden of group B streptococcal and E. coli disease continues," Pediatrics, vol. 127, no. 5, pp. 817-826, 2011.

[11] X.-C. Chen, Y.-F. Yang, R. Wang, H.-F. Gou, and X.-Z. Chen, "Epidemiology and microbiology of sepsis in mainland China in the first decade of the 21st century," International Journal of Infectious Diseases, vol. 31, pp. 9-14, 2015.

[12] S. T. Kizatova and N. I. Dyusembaeva, "Analysis of perinatal causes of infant mortality in the structure of the Karaganda region," Medicine and Ecology, vol. 3, pp. 71-74, 2016.

[13] S. I. Kolesnichenko, A. V. Lavrinenko, I. A. Belyaev et al., "Sensitivity to antibiotics of microorganisms isolated from the blood of resuscitation patients," Clinical Microbiology and Antimicrobial Chemotherapy, vol. 20, p. 25, 2018.

[14] M. H. Tsai, S. M. Chu, J. F. Hsu et al., "Polymicrobial bloodstream infection in neonates: microbiology, clinical characteristics, and risk factors," PLoS One, vol. 9, no. 1, Article ID e83082, 2014.

[15] C. Sakarikou, A. Altieri, S. Minelli, C. Dolfa, M. Piperno, and C. Favalli, "Rapid and cost-effective identification and antimicrobial susceptibility testing in patients with Gram-negative bacteremia directly from blood-culture fluid," Journal of Microbiological Methods, vol. 146, pp. 7-12, 2018.

[16] A. Croxatto, G. Prod'hom, and G. Greub, “Applications of MALDI-TOF mass spectrometry in clinical diagnostic microbiology," FEMS Microbiology Reviews, vol. 36, no. 2, pp. 380-407, 2012.

[17] Clinical and Laboratory Standards Institute (CLSI), Performance Standards for Antimicrobial Disk Susceptibility Tests, Clinical and Laboratory Standards Institute, Wayne, PA, USA, 13 edition, 2018.

[18] The European Committee on Antimicrobial Susceptibility Testing, "Breakpoint tables for interpretation of mics and zone diameters," 2019, http://www.eucast.org/clinical_ breakpoints/.

[19] E. Matuschek, J. Åhman, C. Webster, and G. Kahlmeter, "Antimicrobial susceptibility testing of colistin - evaluation of seven commercial MIC products against standard broth microdilution for Escherichia coli, Klebsiella pneumoniae, Pseudomonas aeruginosa, and Acinetobacter spp," Clinical Microbiology and Infection, vol. 24, no. 8, pp. 865-870, 2018.

[20] A. Tragiannidis, C. Tsoulas, and A. H. Groll, "Invasive candidiasis and candidaemia in neonates and children: update on current guidelines," Mycoses, vol. 58, no. 1, pp. 10-21, 2015.

[21] T. E. Zaoutis, J. Argon, J. Chu, J. A. Berlin, T. J. Walsh, and C. Feudtner, "The epidemiology and attributable outcomes of candidemia in adults and children hospitalized in the United States: a propensity analysis," Clinical Infectious Diseases, vol. 41, no. 9, pp. 1232-1239, 2005.

[22] A. Jain, A. Agarwal, R. K. Verma, S Awasthi, and K. P. Singh, "Intravenous device associated blood stream staphylococcal infection in paediatric patients," The Indian Journal of Medical Research, vol. 134, pp. 193-199, 2011.

[23] L. Santarpia, A. Buonomo, M. C. Pagano et al., "Central venous catheter related bloodstream infections in adult patients on home parenteral nutrition: prevalence, predictive factors, therapeutic outcome," Clinical Nutrition, vol. 35, no. 6, pp. 1394-1398, 2016.

[24] H. M. Abdallah, B. B. Wintermans, E. A. Reuland et al., "Extended-spectrum $\beta$-lactamase- and carbapenemase-producing enterobacteriaceae isolated from Egyptian patients with suspected blood stream infection," PLoS One, vol. 10, no. 5, Article ID e0128120, 2015.

[25] S. Wang, S. Y. Zhao, S. Z. Xiao et al., "Antimicrobial resistance and molecular epidemiology of Escherichia coli causing bloodstream infections in three hospitals in Shanghai, China," PLoS One, vol. 11, no. 1, Article ID e0147740, 2016. 